\title{
The Effect of Solid State Extrusion on Some Physical Properties of High Density Polyethylene
}

\author{
K. Dhawan, ${ }^{*}$ P. C. Jain, and V. S. NANDA \\ Department of Physics \& Astrophysics, University of Delhi, \\ Delhi-110007, India
}

(Received July 27; 1984)

\begin{abstract}
The effect of solid state extrusion on the crystal density of polyethylene has been investigated with the help of wide angle $\mathrm{X}$-ray diffraction studies. It is found that unit cell parameters $a$ - and $b$ - increase with increase in extrusion ratio $R$ while the parameter $c$ - remains practically unaffected. This implies that the crystal density decreases with increasing $R$. We have also determined the change in the degree of crystallinity $X$ as a function of $R$ by X-ray diffraction. It is found that $X$ increases with $R$. Using the usual relation connecting $X$ with the observed specific volume of the semicrystalline polyethylene, the specific volumes of the crystal phase and the amorphous phase, the change in the amorphous specific volume with $R$ has been determined. These results are analysed in terms of Peterlin's model of plastic deformation in semicrystalline polymers and compared with the corresponding reported data for drawn samples. Finally taking account of observed changes in the degree of crystallinity, the crystal and amorphous specific volumes due to extrusion, we have determined the entropy difference $\Delta S_{\mathrm{R}}$, between the unoriented and the oriented amorphous phase as a function of $R$ from the corresponding data for heat of fusion.

KEY WORDS Polyethylene / Solid-State Extrusion / X-Rays / Unit-Cell Dimensions / Crystalline Density / Amorphous Density / Melting / Configurational Entropy /
\end{abstract}

In an earlier paper $^{1}$ we have discussed the melting behavior and morphology of polyethylene (PE) samples extruded in the solid state. There the observed increase in heat of fusion, $H_{\mathrm{f}}$, was attributed to the orientation produced in the amorphous phase. On the other extreme, Kojima et al. $^{2}$ in their solid state extrusion studies have ascribed the increase in $H_{\mathrm{f}}$ solely to the enhancement in the degree of crystallinity. The latter interpretation did not appear justified as it was found that the density of extruded PE does not show an equivalent increase. In our earlier analysis it was, however, tacitly assumed that the crystal and amorphous densities are not affected by extrusion. In order to check on this, X-ray diffraction studies have now been carried out to determine crystal density as a function of extrusion ratio $R$. We have also investigated the changes in the degree of crystallinity $X$ with $R$ through $\mathrm{X}$-ray diffraction studies.

\section{EXPERIMENTAL}

\section{Study of Changes in Crystal Density}

The high density PE used in the present study was of commercial grade, GD 6260 supplied by Hoechst Chemicals Ltd., India. The extrudates were prepared according to the procedures described in our earlier paper. ${ }^{1}$ Wide angle $\mathrm{X}$-ray diffraction pattern of various PE extrudates were taken using a flat film camera. Nickel filtered $\mathrm{Cu}-K_{\alpha}$ radiation (35 $\mathrm{kV}, 15 \mathrm{~mA}$ ) were used for these studies. The incoming beam was collimated with the help of a collimater of length $5 \mathrm{~cm}$ and diameter 0.06

* Present Address: North India Textile Research Association Ghaziabad-201002, India. 
$\mathrm{cm}$. The sample holder was fixed on the collimater and the distance between the specimen and the film was $4 \mathrm{~cm}$. The geometrical constant for the camera setting was obtained by using calcium fluoride diffraction pattern as a standard. Up to the highest extrusion ratio considered in the present study, the extrudates gave the orthorhombic form of diffraction pattern observed for normal PE samples. The unit cell parameters, $a$-, $b$-, and $c$ - were determined using (200), (020) (002) reflections employing the usual relation

$$
d_{h k l}=\frac{1}{(h / a)^{2}+(k / b)^{2}+(l / c)^{2}}
$$

where $d_{h k l}$ is the Bragg's interplanar spacing having the Miller indices $h, k$ and $l$. The broadening in the $\mathrm{X}$-ray diffraction pattern due to the size of the crystallites somewhat limits the accuracy of the results. The error in the values of the cell dimensions was estimated in our work to be about $0.01 \AA$.

\section{Study of Changes in Degree of Crystallinity}

The degree of crystallinity was determined as a function of $R$ by analysing the corresponding $\mathrm{X}$-ray diffractograms. These were obtained with the help of Philips 130 Diffractometer using nickel filtered $\mathrm{Cu}-K_{\alpha}$ radiations from a high intensity $\mathrm{X}$-ray tube $(50 \mathrm{kV}, 30$ $\mathrm{mA})$. X-rays were passed through the sample perpendicular to the flow axis and the intensity of the diffracted beam was recorded on a chart recorder. The degree of crystallinity, neglecting the effect of scattering due to lattice vibrations and imperfections, ${ }^{3}$ was obtained from the relation ${ }^{4}$

$$
X=\frac{A_{\mathrm{c}}}{A_{\mathrm{c}}+K A_{\mathrm{a}}}
$$

where $A_{\mathrm{c}}$ is the area enclosed by the crystalline peaks of intensity versus $2 \theta$ trace $(\theta$ being the angle of diffraction), $A_{\mathrm{a}}$ is the area enclosed by amorphous halo in the same trace and $K$ is a correction factor which takes into account, among other effects, the fact that the scatter- ing intensity of the crystalline reflection has a different angular distribution than the amorphous. In the intensity trace $A_{\mathrm{a}}$ was separated by the normal procedure. ${ }^{5}$ The error in the determination of $X$ was estimated to be about $1 \%$.

\section{RESULTS}

\section{Crystal Density}

The changes in the unit cell parameters $a$ and $b$ - with extrusion ratio $R$ are shown by continuous curves in Figures 1 and 2, respectively, for the sample extruded at $388 \mathrm{~K}$. It may be noted that for small $R, \Delta a[=a(R)-a(1)]$ and $\Delta b[=b(R)-b(1)]$ increase with increasing $R$. At higher $R$, the rate of increase becomes slower till for $R>30$, the increase is hardly

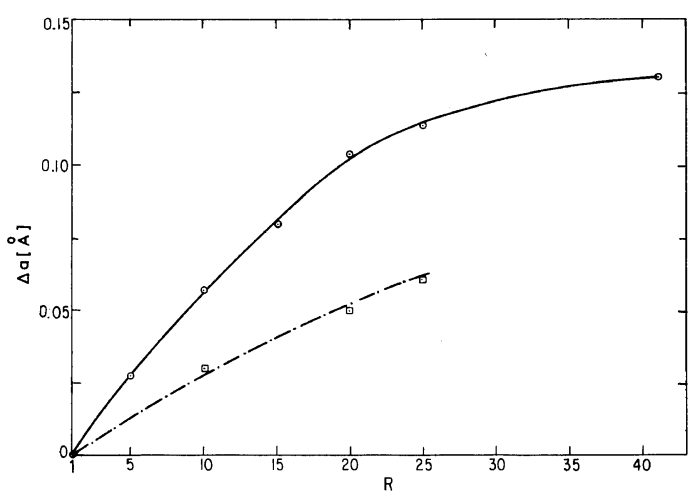

Figure 1. Variation of unit cell parameter $a$ - with extrusion ratio $R$. Full curve (ples; broken curve (-.-.-), drawn PE sample.

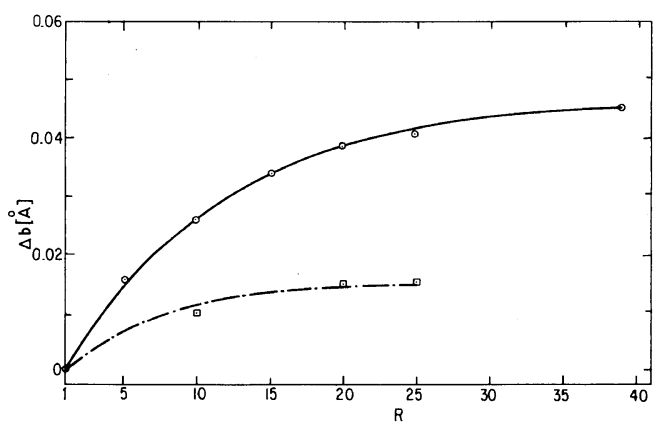

Figure 2. Variation of unit cell parameter $b$ - with extrusion ratio $R$. Full curve (-), extruded PE samples; broken curve (-.-.- ), drawn PE sample. 
perceptible. Our study also indicated that the third dimension $c$ - is practically independent of $R$. In $\mathrm{PE}$ the changes in the crystalline specific volume, $\Delta V_{\mathrm{c}}$, neglecting the second order terms, can be obtained from the relation

$$
\Delta V_{\mathrm{c}}=\frac{N_{\mathrm{A}}}{28} c(b \Delta a+a \Delta b)
$$

where $N_{\mathrm{A}}$ is the Avogadro number. For the calculation of $\Delta V_{\mathrm{c}}$, we used the values $a=$ $7.42 \AA, b=4.94 \AA$, and $c=2.54 \AA$ obtained from our measurements for unextruded sample $(R=1)$. These values are in good agreement with those reported in the literature. ${ }^{6}$

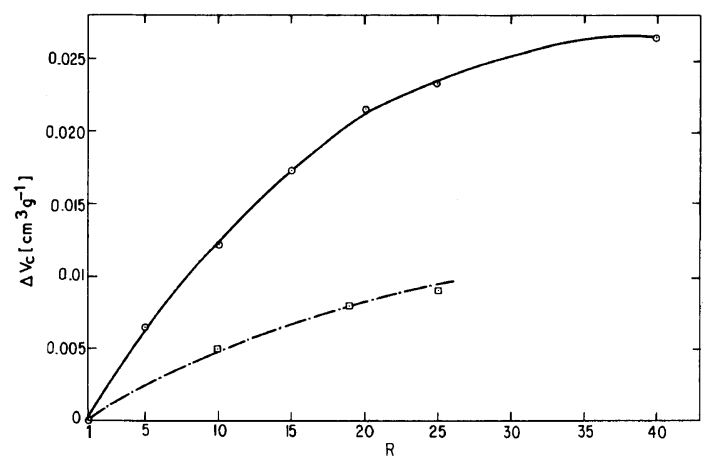

Figure 3. Variation of crystal specific volume $V_{\mathrm{c}}$ with extrusion ratio $R$. Full curve (-), extruded PE samples; broken curve (-.-.-), drawn PE samples.
The changes in $\Delta V_{\mathrm{c}}$ with $R$ calculated from eq 3 for $T=388 \mathrm{~K}$ are shown by the continuous curve in Figure 3. It may be noted that there is a monotonic increase in $\Delta V_{\mathrm{c}}$ with increasing $R$, though for $R>30$ the increase is very small. The variation of $\Delta a$ and $\Delta b$ with extrusion temperature, $T_{\mathrm{E}}$, is shown in Figure 4 for $R=15$. It is seen that $\Delta a$ and $\Delta b$ decrease with increase of $T_{\mathrm{E}}$. In Figure 5, the variation of $\Delta V_{\mathrm{c}}$ with $T_{\mathrm{E}}$ is shown for $R=15$. As could be anticipated, $\Delta V_{\mathrm{c}}$ decreases with increasing $T_{\mathrm{E}}$.

\section{Degree of Crystallinity}

The variation of $\Delta X[=X(R)-X(1)]$ with $R$ for $T_{\mathrm{E}}=388 \mathrm{~K}$ is shown by continuous curve in Figure 6. It may be noted that there is a monotonic increase in $\Delta X$ with $R$, though again for $R>30$ the increase is marginal. The changes in $\Delta X$ with $T_{\mathrm{E}}$ for $R=10$ and $R=15$ are shown in Figure 7 . It is seen that $\Delta X$ increases with increasing $T_{\mathrm{E}}$ for both the $R$ values.

\section{Amorphous Density}

Knowing $V, V_{\mathrm{c}}$ and $X$ for different $R$, the corresponding amorphous specific volume $V_{\text {a }}$ can be determined making use of the usual

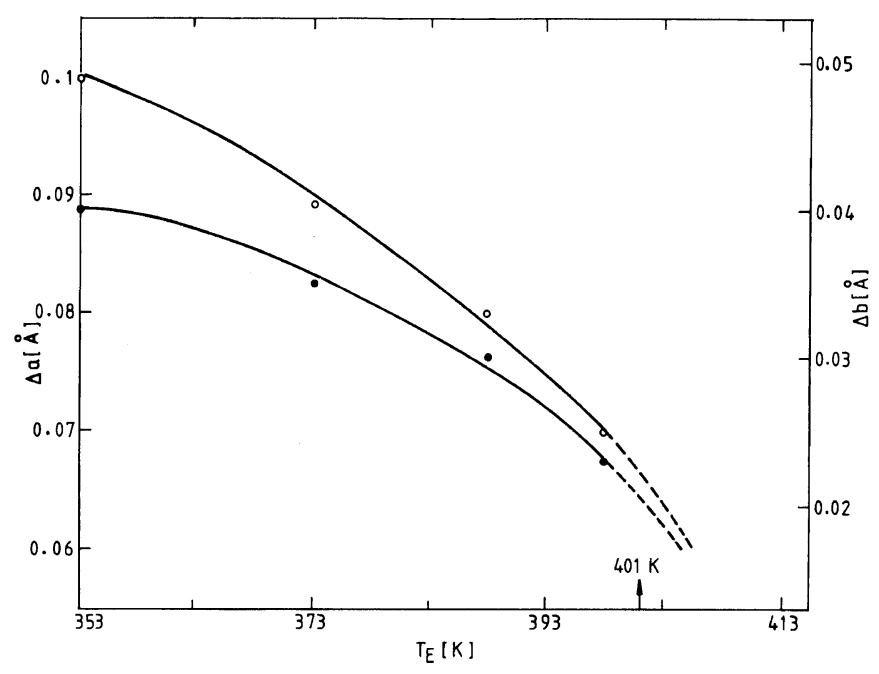

Figure 4. Variation of unit cell parameters $a$ - and $b$ - with extrusion temperature $T_{\mathrm{E}}$ for PE (extrusion ratio $R=15$ ) Upper curve $(\bigcirc), \Delta a$; lower curve $(\bigcirc), \Delta b$. 
K. Dhawan, P. C. JaIn, and V. S. Nanda

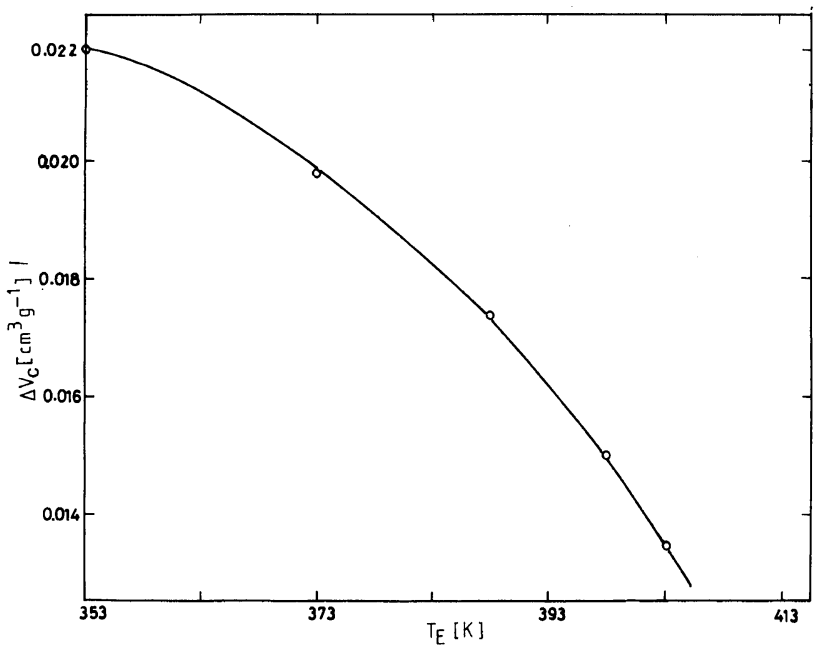

Figure 5. Variation of crystal specific volume $V_{\mathrm{c}}$ with extrusion temperature $T_{\mathrm{E}}$ for PE (extrusion ratio $R=15$ ).

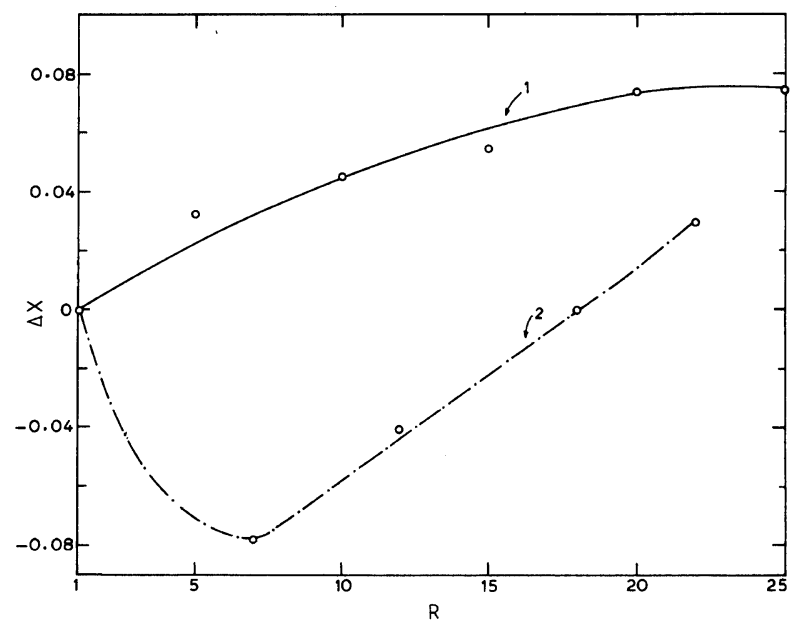

Figure 6. Variation of degree of crystallinity $X$ with extrusion ratio $R$. Curve 1 ( - ), extruded PE samples; curve $2(-\cdot-\cdot-$ ) , drawn PE samples.

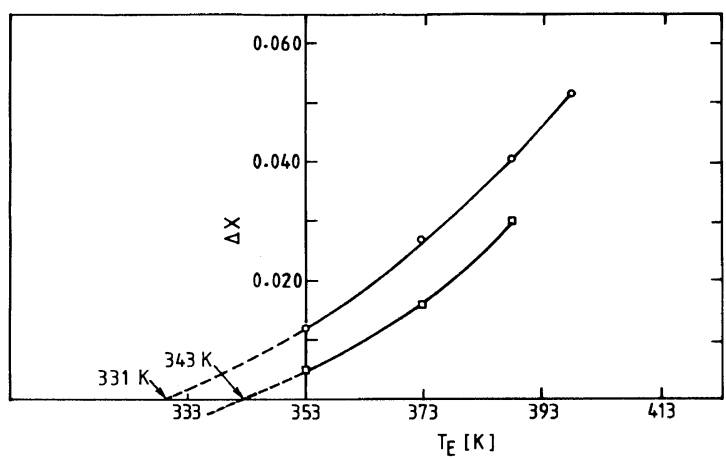

Figure 7. Variation of degree of crystallinity $X$ with extrusion temperature $T_{\mathrm{E}}$ for PE. Upper curve $(\mathrm{O})$, extrusion ratio $R=15$; lower curve $(\square)$, extrusion ratio $R=10$. 


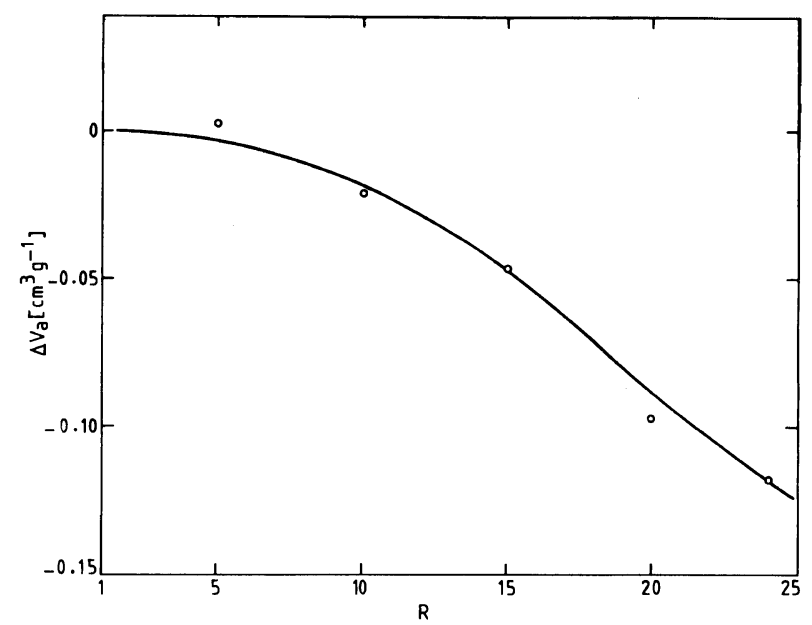

Figure 8. Variation of amorphous specific volume $V_{\mathrm{a}}$ with extrusion ratio $R$ for extruded PE.

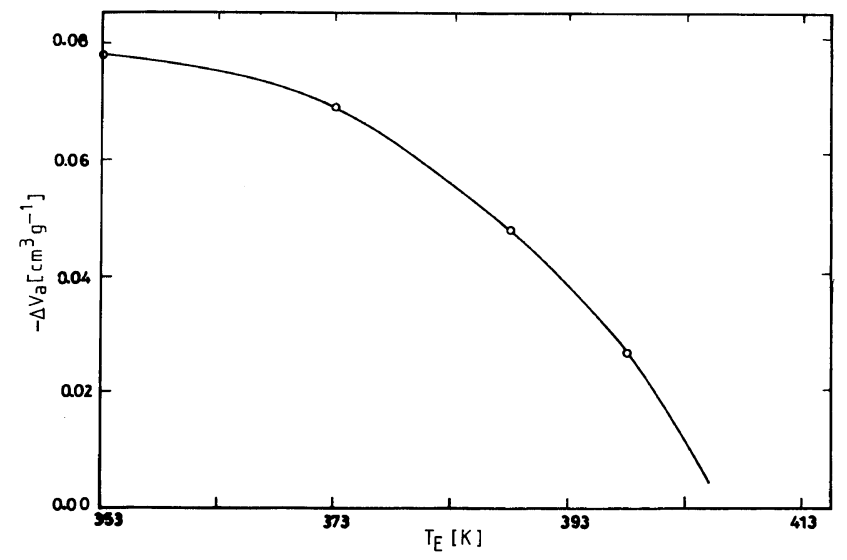

Figure 9. Variation of amorphous specific volume $V_{\mathrm{a}}$ with extrusion temperature $T_{\mathrm{E}}$ for $\mathrm{PE}$ for extrusion ratio $R=15$.

relation

$$
V=X V_{\mathrm{c}}+(1-X) V_{\mathrm{a}}
$$

where $V$ is the specific volume of the sample. The values of $\Delta V_{\mathrm{a}}\left[=V_{\mathrm{a}}(R)-V_{\mathrm{a}}(1)\right]$ obtained from eq 4 for different $R$ at $T_{\mathrm{E}}=388 \mathrm{~K}$ are shown in the form of circles in Figure 8. It may be observed that decrease in $\Delta V_{\mathrm{a}}$ is slow for small $R$ but its variation becomes more rapid for higher values of $R$. The dependence of $\Delta V_{\mathrm{a}}$ on $T_{\mathrm{E}}$ for $R=15$ is shown in Figure 9. It may be noted that $\Delta V_{\mathrm{a}}$ decreases with increase in $T_{\mathrm{E}}$. Similar behavior was also observed for other values of $R$.

\section{DISCUSSION}

According to Peterlin ${ }^{7}$ the plastic deformation due to drawing of semi-crystalline polymers may be considered to have two independent modes. The first, which is important at low deformation ratios, produces the slipping of lamellae in the original material into position of maximum plastic compliance for the chain tilt and slip. This, with increasing deformation ratio, leads to the break up of the 
lamellae into folded chain blocks. Concurrently, due to the second mode, which shows the effects at higher $R$, a new fiber structure appears as the torn off blocks are incorporated into well aligned microfibrils through shear deformation. For PE the molecular rearrangements in the plane perpendicular to the chain axis occur easily because they are opposed by relatively weak van der Waals forces. The shear stresses on adjacent microfibrils bring about changes in the unit cell dimensions $a$ and $b$-. Along the $c$-axis there are strong covalent intermolecular forces which resist deformation. Peterlin's model has been extensively employed to explain the changes in physical properties of drawn samples. In our case during the solid state extrusion, the same two processes of deformation are operative, though shear deformation is expected to play a more dominant role. ${ }^{8}$ As may be noted from Figures 1 and 2, the changes in the unit cell dimensions $a$ - and $b$-for the same deformation ratio, are larger for extruded samples (continuous curves) as compared to drawn samples (dash-dot curves). Consequently, the crystalline specific volume increases with increasing $R$ more steeply in the former case. For example, it may be observed from Figure 3 that for $R=25$ the change in $V_{\mathrm{c}}$ for the extruded sample is more than $2 \%$ whereas for the drawn sample the corresponding change is less than $1 \%{ }^{9}$

The variations of $\Delta a$ and $\Delta b$ with $T_{\mathrm{E}}$ for $R=15$, shown in Figure 4, can be explained on the basis of larger mobility of chains at higher temperature because of which lower shearing stresses are required for flow, thus resulting in smaller changes in unit cell parameters. It may also be observed that even when $T_{\mathrm{E}}$ equals the normal melting point of the processed bullet $\left(T_{\mathrm{m}}^{0}=401 \mathrm{~K}\right)$, the extrapolated curves indicate a change in the unit cell dimensions. Consequently, as is clear from Figure $5, V_{\mathrm{c}}$ of PE deformed at $T_{\mathrm{m}}^{0}$ is different from $V_{\mathrm{c}}$ of undeformed (normal) PE.

\section{Degree of Crystallinity}

It may be noted from curve 1 of Figure 6 that for extruded samples $\Delta X$ increases monotonically with $R$. For comparison, the variation of $\Delta X$ with draw ratio $R$ for polyethylene samples (Marlex 6050 having 82\% initial crystallinity), drawn at $333 \mathrm{~K}$, is also shown (curve 2). These results were obtained by Glentz et al. ${ }^{10}$ through infrared measurements (further details regarding the conditions of drawing are described in their paper). It may be noted that in their case for lower $R$ there is decrease in $\Delta X$ which is followed by an increase for $R>7$. Further, $\Delta X$ values for drawn samples are observed to be always lower than for the extruded samples. The question arises whether the initial decrease in the $\Delta X$ versus $R$ plot is a characteristics of the draw process or it is the effect of processing temperature. This point is discussed in the sequel.

In the present study all extrusions were carried out above $353 \mathrm{~K}$. In this temperature range the mobility of chains is high. The flow process can thus bring about order in the amorphous chains easily through molecular rearrangements. On the other hand, at the draw temperature $(333 \mathrm{~K})$, which is lower by twenty degrees, the mobility of chains is much less. Thus, during the molecular rearrangement, while the change from spherulitic to fibril form is taking place, a part of the material changes from crystalline to amorphous state. When this process is complete $(R>7)$, crystallinity begins to increase for higher $R$ values. In order to demonstrate the effect of processing temperature, we have plotted in Figure $7 \Delta X$ versus $T_{\mathrm{E}}$ for extruded samples $(R=10$ and $R=15)$. It may be noted that in both cases $\Delta X$ increases with increase in $T_{\mathrm{E}}$. Further, at each $T_{\mathrm{E}}, \Delta X$ is higher for higher values of $R$. The extrapolation of these curves on the low temperature side indicates negative $\Delta X$ values for $R=10$ below $343 \mathrm{~K}$. For $R=15$, the corresponding critical value of $T_{\mathrm{E}}$ is $331 \mathrm{~K}$. It is noted that the $\Delta X$ values at $333 \mathrm{~K}$ (corresponding to the experimental draw tempera- 
ture) are $0.2 \times 10^{-2}$ and $-0.4 \times 10^{-2}$ for $R=15$ and $R=10$, respectively. Thus, it seems that the $\Delta X$ versus $R$ variation for the drawn and extruded samples at the same temperature would be qualitatively similar though the actual magnitudes, which depend on the details of the process, can be different.

\section{Amorphous Density}

The $\Delta V_{\mathrm{a}}$ versus $R$ curve shown in Figure 8 indicates that amorphous density $\rho_{\mathrm{a}}\left(=1 / V_{\mathrm{a}}\right)$ increases with deformation ratio $R$. During the extrusion process; chains in the amorphous phase get aligned in the flow direction. This alignment reduces the average separation between them and results in an increase in amorphous density. In Figure 9, the variation of $\Delta V_{\mathrm{a}}$ with $T_{\mathrm{E}}$ is shown for $R=15$. It is observed that amorphous density of the sample extruded at higher temperature is closer to the amorphous density of the normal material. Since the ordering effects in the amorphous phase due to extrusion are opposed by thermal agitation to a greater extent at higher temperature, the extent of alignment achieved at the same $R$ value is lower which results in smaller density increase.

\section{Melting Behavior}

On extruding or drawing a sample, since the crystallinity, the crystal density as well as the amorphous density are affected, the analysis of the melting behavior becomes quite complex. The heat of transformation $H_{\mathrm{f}}$ of the oriented semi-crystlline polymer to the melt can be written in the form

$$
H_{\mathrm{f}}=X H_{\mathrm{fc}}+(1-X) H_{\mathrm{fa}}
$$

where $X$ is the crystallinity of the deformed sample, $H_{\mathrm{fc}}$ and $H_{\mathrm{fa}}$ are respectively the heats of transformation of the oriented crystal and the oriented amorphous phases. For unoriented semi-crystalline polymer, the heat of transformation is given by

$$
H_{\mathrm{f}}^{0}=X^{0} H_{\mathrm{fc}}^{0}
$$

where $X^{0}$ is the crystallinity of normal polymer and $H_{\mathrm{fc}}^{0}$ is the heat of transformation of the normal crystal phase. The increase in heat of fusion is given by

$$
\begin{aligned}
H_{\mathrm{f}}= & H_{\mathrm{f}}-H_{\mathrm{f}}^{0}=(1-X) H_{\mathrm{fa}} \\
& +X\left(H_{\mathrm{fc}}-H_{\mathrm{fc}}^{0}\right)+\Delta X H_{\mathrm{fc}}^{0}
\end{aligned}
$$

where $\Delta X=X-X^{0}$. As far as $H_{\mathrm{fa}}$ is concerned, it may be regarded as made up of (i) the heat used for the configurational entropy increase by the amount $\Delta S_{\mathrm{R}}$ and (ii) the heat utilized in isothermal decrease of density which involves change in internal energy. Thus we write

$$
H_{\mathrm{fa}}=T_{\mathrm{m}} \Delta S_{\mathrm{R}}+U_{\mathrm{a}}^{0}\left(T_{\mathrm{m}}, V_{\mathrm{a}}^{0}\right)-U_{\mathrm{a}}\left(T_{\mathrm{m}}, V_{\mathrm{a}}\right)
$$

where $U_{\mathrm{a}}^{0}$ and $U_{\mathrm{a}}$ denote the internal energies of the normal amorphous sample and the oriented amorphous sample respectively. Expanding $U_{\mathrm{a}}\left(T_{\mathrm{m}}, V_{\mathrm{a}}\right)$ in the neighborhood of $V_{\mathrm{a}}^{0}$, we get

$$
U_{\mathrm{a}}\left(T_{\mathrm{m}}, V_{\mathrm{a}}\right)-U_{\mathrm{a}}^{0}\left(T_{\mathrm{m}}, V_{\mathrm{a}}^{0}\right)+V_{\mathrm{a}} T_{\mathrm{m}} E_{\mathrm{a}}^{0} \gamma_{\mathrm{a}}^{0}
$$

where $E_{\mathrm{a}}^{0}$ and $\gamma_{\mathrm{a}}^{0}$ are respectively the bulk modulus and the coefficient of thermal expansion of the normal amorphous phase.

For the crystal phase, the contribution of the change in configurational entropy of the crystallites due to the effect of extrusion (or drawing) is expected to be very small, we may then write to a good approximation

$$
\begin{aligned}
H_{\mathrm{fc}}-H_{\mathrm{fc}}^{0} & =\left\{U_{\mathrm{c}}^{0}\left(T_{\mathrm{m}}^{0}, V_{\mathrm{c}}^{0}\right)-U_{\mathrm{c}}\left(T_{\mathrm{m}}, V_{\mathrm{c}}\right)\right\} \\
& -\left\{U_{\mathrm{a}}^{0}\left(T_{\mathrm{m}}^{0}\right)-U_{\mathrm{a}}^{0}\left(T_{\mathrm{m}}\right)\right\}
\end{aligned}
$$

where $U_{\mathrm{c}}^{0}$ and $U_{\mathrm{c}}$ are internal energies of normal (undeformed) crystal and oriented (deformed) crystal. It may be noted that in eq 9 both the internal energy terms for the amorphous phase have the same superscript and refer to the normal (undeformed) state. Expanding $U_{\mathrm{c}}\left(T_{\mathrm{m}}, V_{\mathrm{c}}\right)$ about the normal melting temperature $T_{\mathrm{m}}^{0}$ and normal crystal specific volume, $V_{\mathrm{c}}^{0}$, we get

$$
\begin{aligned}
& U_{\mathrm{c}}\left(T_{\mathrm{m}}, V_{\mathrm{c}}\right)=U_{\mathrm{c}}^{0}\left(T_{\mathrm{m}}^{0}, V_{\mathrm{c}}^{0}\right)+\Delta T_{\mathrm{m}} C_{\mathrm{vc}}^{0} \\
& \quad+\Delta V_{\mathrm{c}} T_{\mathrm{m}}^{0} E_{\mathrm{c}}^{0} \gamma_{\mathrm{c}}^{0}
\end{aligned}
$$




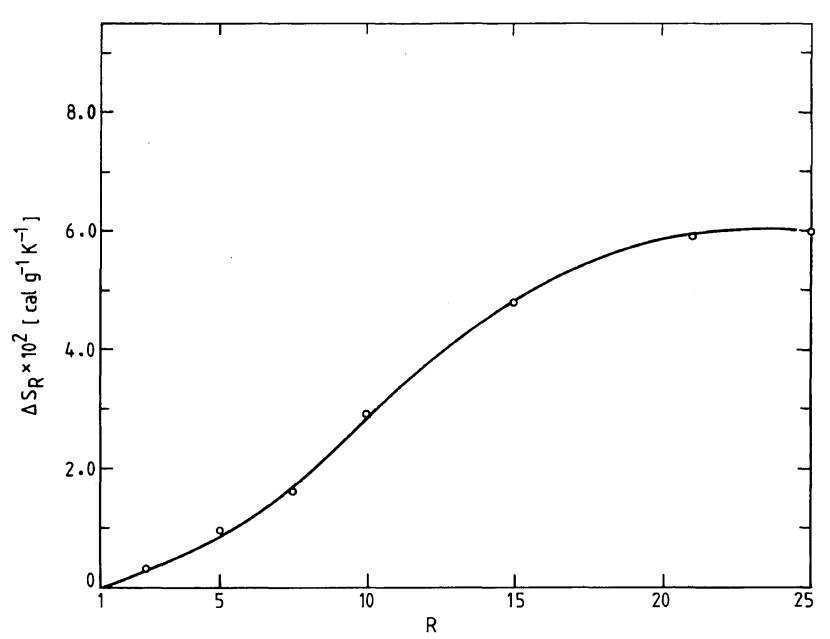

Figure 10. Variation of entropy difference $\Delta S_{\mathrm{R}}$ with extrusion ratio $R$ for the amorphous part of semicrystalline PE.

where $\Delta T_{\mathrm{m}}=T_{\mathrm{m}}-T_{\mathrm{m}}^{0}$ and $\Delta V_{\mathrm{c}}=V_{\mathrm{c}}-V_{\mathrm{c}}^{0}$ where $V_{\mathrm{c}}$ and $V_{\mathrm{c}}^{0}$ are the specific volumes of the crystal in the deformed and undeformed states respectively. In eq $9 \mathrm{a}, C_{\mathrm{vc}}^{0}$ is the specific heat of normal crystal at constant volume while $E_{\mathrm{c}}^{0}$ and $\gamma_{\mathrm{c}}^{0}$ are respectively the bulk modulus and the coefficient of thermal expansion of the normal crystal at $T_{\mathrm{m}}^{0}$. Similarly for the amorphous phase, we may write

$$
U_{\mathrm{a}}^{\mathrm{o}}\left(T_{\mathrm{m}}\right)=U_{\mathrm{a}}^{\mathrm{o}}\left(T_{\mathrm{m}}^{0}\right)+\Delta T_{\mathrm{m}} C_{\mathrm{va}}^{0}
$$

where $C_{\mathrm{va}}^{0}$ is the specific heat of the amorphous phase. Substituting from eq 7, 8, 9a, and $9 \mathrm{~b}$ in eq 6 , we get the expression

$$
\begin{aligned}
\Delta S_{\mathrm{R}}= & \frac{1}{T_{\mathrm{m}}(1-X)}\left[\Delta H_{\mathrm{f}}+X\left\{T_{\mathrm{m}}\left(C_{\mathrm{vc}}^{0}-C_{\mathrm{va}}^{0}\right)\right.\right. \\
& \left.+\Delta V_{\mathrm{c}} T_{\mathrm{m}}^{0} E_{\mathrm{c}}^{0} \gamma_{\mathrm{c}}^{0}\right\} \\
& \left.+(1-X) \Delta V_{\mathrm{a}} E_{\mathrm{m}}^{0} E_{\mathrm{a}}^{0} \gamma_{\mathrm{a}}^{0}-\Delta X H_{\mathrm{fc}}^{0}\right]
\end{aligned}
$$

For the evaluation of $\Delta S_{\mathrm{R}}$ from eq 10 we require some characteristics of the crystal and the amorphous phases of the normal and deformed samples. The values of $T_{\mathrm{m}}^{0}, \Delta T_{\mathrm{m}}$, and $\Delta H_{\mathrm{f}}$ have been reported in our earlier paper, ${ }^{1}$ while in this paper $X, \Delta X, \Delta V_{\mathrm{c}}$, and $\Delta V_{\mathrm{a}}$ results have been given. The evaluation of the bulk modulus, the coefficient of thermal ex- pansion and the specific heat at constant volume requires a knowledge of the equation of state. For the crystal using the equation state of Nanda et al., ${ }^{12}$ we find at $404 \mathrm{~K}$, $\gamma_{\mathrm{c}}^{0}=3.12 \times 10^{-4} \mathrm{~K}^{-1}$ and $E_{\mathrm{c}}^{0}=49.82 \mathrm{kbar}$. Using these values and the experimental value of $C_{\mathrm{pc}}^{013}$ in the thermodynamic result

$$
C_{\mathrm{pc}}^{0}-C_{\mathrm{vc}}^{0}=\gamma_{\mathrm{c}}^{0} E_{\mathrm{c}}^{0} T V_{\mathrm{c}}^{0}
$$

we get $C_{\mathrm{vc}}^{0}=0.564 \mathrm{calg}^{-1} \mathrm{~K}^{-1}$. For the amorphous phase, values of $E_{\mathrm{a}}^{0}$ and $\gamma_{\mathrm{a}}^{0}$ can be obtained by making use of Simha and Wilson's equation of state, ${ }^{14}$ while $C_{\mathrm{va}}^{0}$ is determined from $C_{\mathrm{pa}}^{0}$ in the same way as for the crystalline phase. At $404 \mathrm{~K}$ we find $\gamma_{\mathrm{a}}^{0}=7.44 \times 10^{-4} \mathrm{~K}^{-1}$, $E_{\mathrm{a}}^{0}=10.93 \mathrm{kbar}$, and $C_{\mathrm{va}}^{0}=0.665 \mathrm{cal} \mathrm{g}^{-1} \mathrm{~K}^{-1}$. The $\Delta S_{\mathrm{R}}$ results obtained from eq 10 are plotted as a function of $R$ in Figure 10. It is observed that $\Delta S_{\mathrm{R}}$ increases with $R$ though for $R>20$ the variation is very small due to saturation effect.

Acknowledgements. The authors acknowledge with thanks the U.N.D.P. assistance in the form of certain items of equipment. This work was supported by a research grant received from the University Grants Commission (India). 


\section{REFERENCES}

1. K. Dhawan, D. R. Chaubey, Y. S. Yadav, P. C. Jain, and V. S. Nanda, Polym. J., 12, 411 (1980).

2. S. Kojima, C. R. Desper, and R. S. Porter, J. Polym. Sci.; Polym. Phys., Ed., 16, 1721 (1978).

3. W. Ruland, Acta Cryst., 14, 1180 (1961).

4. B. Wunderlich, "Macromolecular Physics," Vol. 1, Academic Press, New York, 1973, p 398.

5. M. Kukuda and R. Ullmann, J. Polym. Sci., 45, 91 (1960); see also P. H. Hermans and A. Weidinger, Macromol. Chem., 44, 34 (1961).

6. G. T. Davis, R. K. Eby, and J. P. Colson, J. Appl. Phys., 41, 4316 (1970).

7. A. Peterlin, J. Mater. Sci., 6, 490 (1971).
8. A. G. Kolback and D. R. Ulhamann, J. Polym. Sci., Polym. Phys., Ed., 15, 27 (1977).

9. W. Glentz, N. Morosoff, and A. Peterlin, J. Polym. Sci., Polym. Lett., 9, 211 (1977).

10. G. Glentz and A. Peterlin, J. Macromol. Sci. Phys., B4, 473 (1970).

11. A. Ciferre, C. A. J. Hoeve, and P. J. Flory, J. Am. Chem. Soc., 83, 1015 (1961).

12. V. S. Nanda, O. P. Pahuja, and F. C. Auluck, $J$. Macromol. Sci., B18, 393 (1980).

13. B. Wunderlich and H. Bauer, Adv. Polym. Sci., 7, 151 (1970).

14. R. Simha and P. S. Wilson, Macromolecules, 6, 908 (1973). 